\title{
Review Article \\ From Molecular Classification to Targeted Therapeutics: The Changing Face of Systemic Therapy in Metastatic Gastroesophageal Cancer
}

\author{
Adrian Murphy ${ }^{1}$ and Ronan J. Kelly ${ }^{1,2}$ \\ ${ }^{1}$ Upper Aerodigestive Malignancies Division, Sidney Kimmel Comprehensive Cancer Center at Johns Hopkins, \\ Baltimore, MD 21231, USA \\ ${ }^{2}$ Gastroesophageal Cancer Therapeutics Program, Sidney Kimmel Comprehensive Cancer Center at Johns Hopkins, \\ Bunting Blaustein Cancer Research Building, 1650 Orleans Street, Room G93, Baltimore, MD 21231, USA
}

Correspondence should be addressed to Ronan J. Kelly; rkelly25@jhmi.edu

Received 10 November 2014; Accepted 15 January 2015

Academic Editor: Antoni Castells

Copyright (C) 2015 A. Murphy and R. J. Kelly. This is an open access article distributed under the Creative Commons Attribution License, which permits unrestricted use, distribution, and reproduction in any medium, provided the original work is properly cited.

\begin{abstract}
Histological classification of adenocarcinoma or squamous cell carcinoma for esophageal cancer or using the Lauren classification for intestinal and diffuse type gastric cancer has limited clinical utility in the management of advanced disease. Germline mutations in E-cadherin ( $\mathrm{CDH1}$ ) or mismatch repair genes (Lynch syndrome) were identified many years ago but given their rarity, the identification of these molecular alterations does not substantially impact treatment in the advanced setting. Recent molecular profiling studies of upper GI tumors have added to our knowledge of the underlying biology but have not led to an alternative classification system which can guide clinician's therapeutic decisions. Recently the Cancer Genome Atlas Research Network has proposed four subtypes of gastric cancer dividing tumors into those positive for Epstein-Barr virus, microsatellite unstable tumors, genomically stable tumors, and tumors with chromosomal instability. Unfortunately to date, many phase III clinical trials involving molecularly targeted agents have failed to meet their survival endpoints due to their use in unselected populations. Future clinical trials should utilize molecular profiling of individual tumors in order to determine the optimal use of targeted therapies in preselected patients.
\end{abstract}

\section{Introduction}

While the incidence of gastric cancer is decreasing in the United States, the rates of esophageal cancer are increasing. In 2014 it is anticipated that 22,220 and 18,170 patients will be newly diagnosed with gastric cancer and esophageal cancer, respectively, while 26,440 men and women will die as a result of upper gastrointestinal (GI) tumors [1]. Gastroesophageal cancer remains endemic in many parts of the world with an estimated new cancer incidence of $1,471,000$ or $11.6 \%$ of the global cancer burden and a death rate annually of $1,144,000$ people or $15.1 \%$ of cancer-related deaths worldwide. Taken together, esophageal cancer and gastric cancer are second only to lung cancer in incidence and in mortality [2]. Despite these figures, there has been a dearth of scientific breakthroughs in these tumor types which have resulted in significant survival advantages.

The location of upper GI tumors in western countries has changed dramatically in recent years. Distal gastric cancer, which previously predominated, has become uncommon, whereas the incidence of tumors of the gastric cardia and gastroesophageal junction has increased dramatically. Prior to the 1970s, distal esophageal adenocarcinomas were uncommon, representing $0.8-3.7 \%$ of esophageal cancers [3]. Over the past three decades, there has been a sevenfold increase in the incidence of esophageal adenocarcinoma among US white males, now accounting for more than half of the cases of esophageal cancer. Through the 1980s, the increases in the rates of these tumors have been on the order of $5-10 \%$ per year, a faster pace than for virtually any other 
TABLE 1: Current and recently completed phase III trials in gastric and gastroesophageal junction cancer.

\begin{tabular}{|c|c|c|c|c|}
\hline Agent & Clinical trial & Randomization & $n$ & NCT identifier \\
\hline \multicolumn{5}{|c|}{ HER2 inhibitors } \\
\hline Pertuzumab & JACOB & Pertuzumab in combination with Herceptin and chemotherapy & 780 & NCT01774786 \\
\hline T-DM1 & GATSBY & T-DM1 with or without taxane. & 412 & NCT01641939 \\
\hline Trastuzumab & HELOISE & XP-T (standard versus high-dose) & 400 & NCT01450696 \\
\hline \multicolumn{5}{|c|}{ MET pathway inhibitors } \\
\hline Rilotumumab & RILOMET-2 & $\mathrm{XP}$ with or without rilotumumab & 450 & NCT02137343 \\
\hline Rilotumumab & RILOMET-1 & ECX with or without rilotumumab & 600 & NCT01697072 \\
\hline Onartuzumab & METGASTRIC & FOLFOX with or without onartuzumab & 800 & NCT01662869 \\
\hline \multicolumn{5}{|c|}{ Cancer stemness inhibitor } \\
\hline BBI608 & BRIGHTER & Paclitaxel with or without BBI608 & 680 & NCT02178956 \\
\hline \multicolumn{5}{|c|}{ EGFR inhibitors } \\
\hline Panitumumab & REAL-3 & EOX with or without panitumumab & 574 & NCT00824785 \\
\hline Cetuximab & EXPAND & $\mathrm{XP}$ with or without cetuximab & 904 & NCT00678535 \\
\hline \multicolumn{5}{|c|}{ Angiogenesis inhibitors } \\
\hline Ramucirumab & REGARD & Ramucirumab versus BSC & 355 & NCT00917384 \\
\hline Ramucirumab & RAINBOW & Paclitaxel with or without ramucirumab & 665 & NCT01170663 \\
\hline Regorafenib & INTEGRATE & Regorafenib versus BSC & 150 & Actrn12612000239864 \\
\hline
\end{tabular}

T-DM1: trastuzumab emtansine; XP: cisplatin, capecitabine; XP-T: cisplatin, capecitabine; T: trastuzumab; ECX: epirubicin, cisplatin, and capecitabine; FOLFOX: 5FU, folinic acid, and oxaliplatin; EOX: epirubicin, oxaliplatin, and capecitabine; BSC: best supportive care.

cancer in the United States [3]. This has been attributed to declining chronic infection rates by Helicobacter pylori and an increased incidence of gastroesophageal reflux disease and obesity [4-6].

\section{Cytotoxic Chemotherapy for Gastroesophageal Cancer}

The treatment of metastatic gastroesophageal junction and gastric cancer has been poorly investigated and at present there is no single chemotherapeutic regimen that is considered standard first-line treatment. Various combinations of platinum-based doublets and triplets have been prescribed depending on patient performance status and physician preference. Current therapy for advanced disease is limited and survival rarely exceeds one year despite aggressive treatment with modern chemotherapy. Second-line regimens have, until recently, been considered ineffective. A number of different drugs (e.g., alkylating agents, platinum compounds, $5 \mathrm{FU}$, and taxanes) are available for the treatment of gastroesophageal cancer but no means of selecting therapy based upon the biology of the tumor is currently available (Table 1). HER2 status remains the only validated molecular marker which influences clinician decision-making in the metastatic setting. At present the combination of a platinum and fluorouracil, either alone or in combination with a third drug such as epirubicin or a taxane, constitutes the most effective treatment option in the first-line metastatic setting [7]. Standard first-line options include DCF (docetaxel, cisplatin, and $5 \mathrm{FU}$ ), ECF/EOX (epirubicin, cisplatin/oxaliplatin, and $5 \mathrm{FU} /$ capecitabine), or FOLFOX (5 FU, oxaliplatin) [8-10]. Additional FDA approved 2nd line agents include docetaxel, paclitaxel, and irinotecan [11-14].

\section{Molecular Classification of Gastric Cancer}

Molecular profiling studies have been performed using gene expression or DNA sequencing and have identified distinctive molecular signatures which may predict responsiveness to systemic therapies. Earlier studies in gastric cancer concentrated on molecular signatures which characterized the processes of tumorigenesis. Microarray-based gene expression profiling identified characteristic expression patterns which readily discern premalignant from malignant tissues [15]. Chronic gastritis tissue expressed a marked mitochondrial gene expression signature possibly as a response to $H$. pylori infection, for example, NDUF (NADH dehydrogenase), whereas intestinal metaplastic tissue expressed a more transformed phenotype including many intestinal differentiation genes which were not expressed in tumor tissues, for example, CDX1, MYO1A, and villin A. Later studies examined whether the molecular signature could predict sensitivity to chemotherapy [16]. Genomic subtypes (intestinal and diffuse) identified from in vitro studies in gastric cancer and validated in primary tumors were found to be prognostic of survival and had the ability to predict sensitivity to $5 \mathrm{FU}$ and/or platinum agents. It was possible to detect these subtypes by immunohistochemical analysis of LGALS4 and CDH17 expression. These studies may ultimately identify predictive biomarkers allowing physicians to personalize chemotherapy selection in gastric cancer.

Molecular profiling has been extended in an attempt to predict responsiveness to targeted therapies [17]. Gene expression patterns were analyzed with advanced bioinformatics tools to identify molecular signature subtypes which predicted response to inhibitors of the PI3K/Akt/mTOR pathway. 
The Cancer Genome Atlas Research Network (TCGA) has recently performed a comprehensive molecular characterization of gastric tumors from 295 patients who had not been treated with prior chemotherapy or radiotherapy [18]. Detailed genetic analysis was performed using array-based somatic copy number analysis, whole-exome sequencing, array-based DNA methylation profiling, mRNA sequencing, microRNA sequencing, and reverse-phase protein arrays. It has proposed four subtypes (Figure 1(a)): (1) tumors positive for Epstein-Barr virus, (2) microsatellite unstable tumors, (3) genomically stable tumors, and (4) tumors with chromosomal instability.

EBV-associated tumors were shown to have a higher prevalence of DNA hypermethylation than any other tumor reported by the TCGA. All EBV-positive tumors displayed CDKN2A promoter hypermethylation and $80 \%$ had PIK3CA mutations. In addition, PD-L1/2 expression was elevated in EBV-positive tumors suggesting a role of targeted immunotherapy in this subset of gastric tumors. Microsatellite unstable (MSI) tumors generally lacked targetable amplifications although mutations in PIK3CA, HER2, HER3, and EGFR were noted. BRAF (V600E) mutations were not seen in gastric MSI tumors unlike its counterpart in colorectal cancer. Genomically stable gastric tumors are enriched for the diffuse histological variant and have newly described mutations in RHOA which acts through several effectors to control actinmyosin-dependent cell contractility and motility. In addition, a recurrent interchromosomal translocation (between CLDN18 and ARHGAP26) implicated in cell motility was found in genomically stable gastric tumors. Almost half of gastric tumors demonstrated chromosomal instability which was characterized by marked aneuploidy and focal amplification of receptor tyrosine kinases such as amplification of VEGFA and frequent amplifications of cell cycle mediators (CCNE1, CCND1, and CDK6). This study has considerably added to our knowledge of the molecular subtyping of gastric cancer and hopefully will permit improved patient selection for clinical trials in the future.

\section{Molecular Classification of Esophageal Cancer}

Chromosomal aberrations leading to gene dysregulation have been reported in esophageal cancer including amplifications on $8 \mathrm{q}$ and $17 \mathrm{q}$ mapped to the $C-M Y C$ and $E R B B 2$ oncogenes $[19,20]$.

The role of $M Y C$ in the pathogenesis of esophageal cancer is not well defined and additional research is required. Loss of heterozygosity of TP53 occurs in greater than $50 \%$ of cases of esophageal cancer and is considered a strong predictor of disease progression [21-23]. In addition, two genes reported to have homozygous deletions in esophageal cancer are p16/CDKN2A and FHIT [24]. Abeloff et al. performed an integrative analysis of array-comparative genomic hybridization and matched gene expression profiling to reveal novel genes with prognostic significance in esophageal adenocarcinomas [25]. The authors identified 17 common regions $(>5 \%)$ of gain and 11 common regions of losses in 56 resected specimens with associated long-term clinical follow-up data. Novel regions identified included loci 11p13 and 21q21.2.

Genes with high copy number and expression correlations included two deletions ( $p 16 / C D K N 2, M B N L 1)$ and four gains (EGFR, WT1, NEIL2, and MTMR9). These genes individually $(P<0.06)$ and collectively had prognostic significance $(P=0.008)$. A host of additional genes have been studied for mutations in esophageal cancer, but in most of these single gene studies, very few mutations have been identified. In an effort to perform a comprehensive evaluation of all coding regions for mutations, Agrawal et al. performed a comprehensive study of esophageal cancer exomes including both adenocarcinomas and squamous cell carcinomas [26]. Inactivating mutations of NOTCH1 were identified in $21 \%$ of esophageal squamous cell carcinomas but not in adenocarcinomas.

Dulak et al. conducted an analysis of somatic copy-number alterations using high-density genomic profiling arrays in 296 esophageal and gastric cancers [27]. Amplified genes were noted in $37 \%$ of gastric/esophageal tumors, including ERBB2, FGFR1, FGFR2, EGFR, and MET, suggesting that some of these may be viable targets in esophageal cancer although amplification of some of these may be more prevalent in gastric tumors.

\section{Molecular Targets in Advanced Disease}

5.1. HER2. HER2 is a protooncogene which is encoded by $E R B B 2$ found on chromosome 17. It belongs to the HER family of membrane-bound receptor tyrosine kinases which is responsible for the initiation of cell signaling pathways via phosphoinositide 3-kinase, phospholipase $\mathrm{C}$, and mitogenactivated protein kinase [28]. While originally known for its effects in breast cancer, HER2 overexpression has been shown to result in worse prognosis in gastric cancer $[29,30]$ although there are conflicting studies which suggest that it has no effect or is even beneficial in terms of prognosis [31,32].

The TOGA trial was a phase III prospective trial which demonstrated the benefits of adding trastuzumab, a humanized monoclonal antibody targeting HER2, to a platinumbased doublet in the presence of HER2 IHC 2+ or FISH amplified metastatic gastroesophageal or gastric cancer [33]. In this trial, 594 patients were randomly assigned to study treatment (trastuzumab plus chemotherapy, $n=298$; chemotherapy alone, $n=296$ ), of whom 584 were included in the primary analysis $(n=294 ; n=290)$. Median overall survival was 13.8 months (95\% CI 12-16 months) in those assigned to trastuzumab plus chemotherapy compared with 11.1 months in those assigned to chemotherapy alone (hazard ratio 0.74 ; 95\% CI $0.60-0.91 ; P=0.0046)$. Unfortunately only $15-20 \%$ of patients with esophageal or gastroesophageal tumors are HER2 positive [32] and intestinal-type disease and tumors at the gastroesophageal junction and proximal stomach have higher HER2 expression [33]. The benefit of trastuzumab was confined to those with IHC $2+/ 3+$ and FISH positivity.

The practice of maintenance trastuzumab and continuing trastuzumab until evidence of disease progression are commonplace in the management of breast cancer [34, 35] but there is a lack of data indicating that this is a successful 


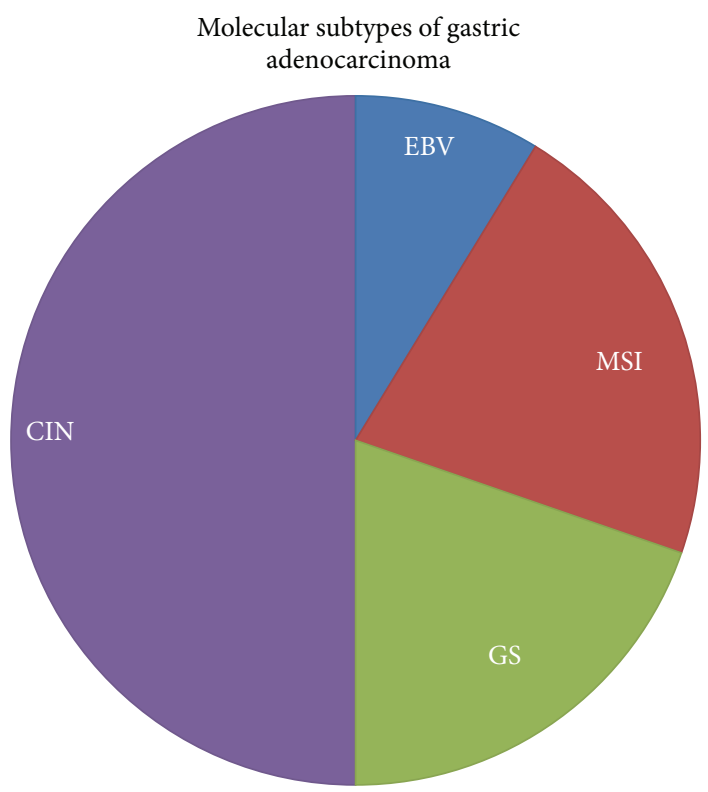

(a)

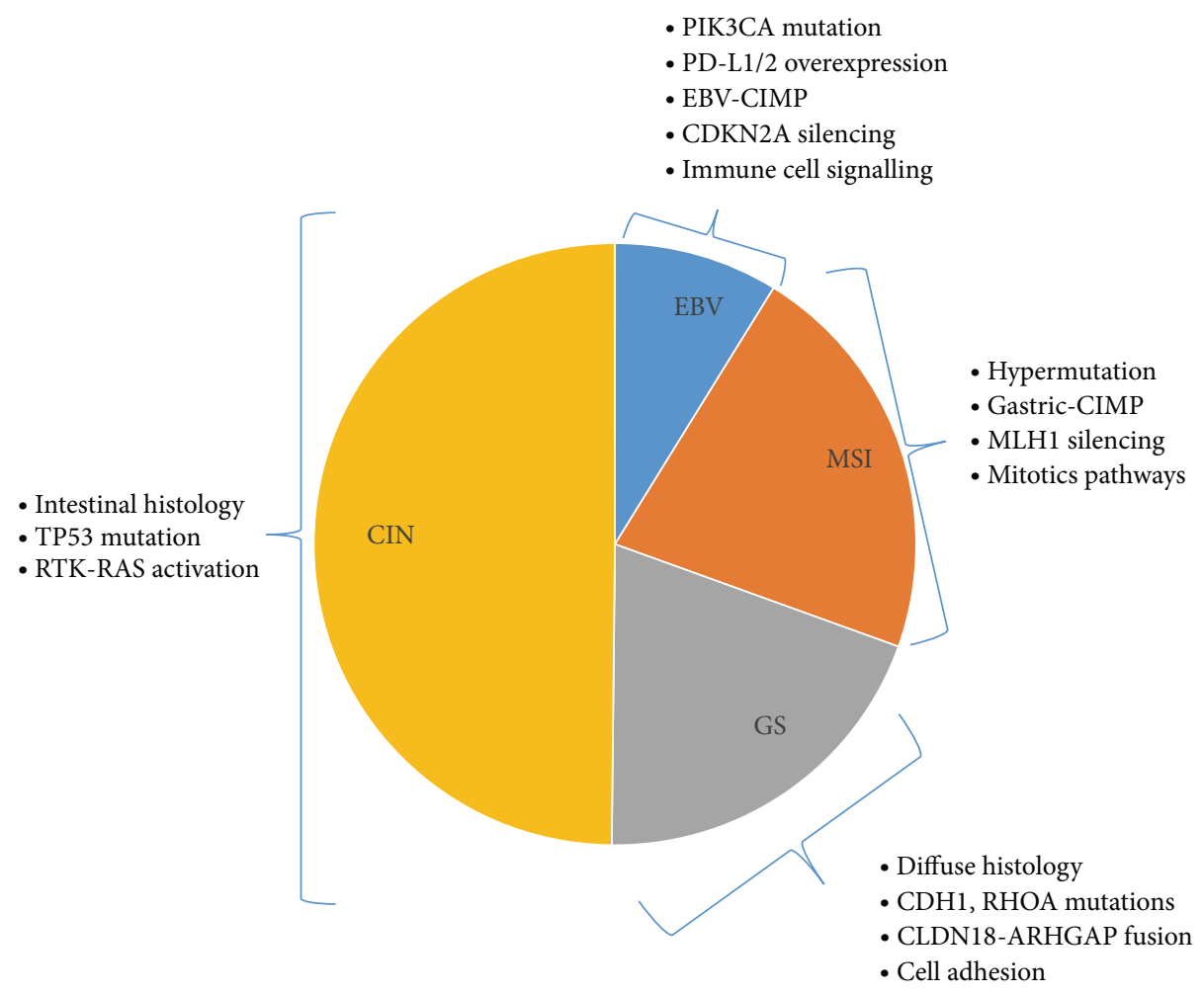

(b)

FIGURE 1: (a) Molecular classification of gastric adenocarcinomas. Primary gastric adenocarcinomas $(n=295)$ were analyzed in the TCGA project and found to have four main subtypes: CIN (chromosomal instability) 49.8\%, GS (genomically stable) 19.6\%, MSI (microsatellite instability) $21.7 \%$, and EBV (Epstein-Barr virus), positive 8.8\%. Adapted from data in TCGA [18]. (b) Characteristics of molecular subtypes of gastric cancer. Adapted from data in TCGA [18]. The key features of each molecular subtype are listed adjacent to the representation of subtype. 
strategy in gastroesophageal cancer although a Japanese trial is currently investigating this approach [36]. The development of resistance to trastuzumab has prompted investigating alternative drugs which target HER2. Lapatinib is an oral dual tyrosine kinase inhibitor which targets EGFR and HER2 domains resulting in blocked autophosphorylation and downstream signaling [37]. The phase III TYTAN study compared paclitaxel with or without lapatinib in HER2 positive gastric cancer in the second-line setting in Asian patients [38]. Median overall survival was 11 months with paclitaxel plus lapatinib compared to 8.9 months with paclitaxel alone $(P=0.1044)$. There was also no significant difference in PFS or TTP. Pertuzumab is a monoclonal antibody which binds to HER2 preventing its dimerization with other HER receptors [39]. It is currently the subject of a phase III trial (JACOB) comparing pertuzumab/trastuzumab + chemotherapy to pertuzumab + chemotherapy [40]. Trastuzumab emtansine (TDM1) is an antibody-drug conjugate combining trastuzumab and DM1 which is a cytotoxic/microtubule polymerization agent [41]. T-DM1 binds to the HER2 receptor resulting in internalization of the DM1-receptor complex which results in the inhibition of cell division/growth and ultimately cell death [42]. The ongoing GATSBY trial is currently investigating T-DM1 versus a taxane in patients with previously treated HER2-positive metastatic or locally advanced gastric cancer [43].

5.2. EGFR. The epidermal growth factor receptor (EGFR) is known to play an important role in the initiation of signaling transduction cascades via phosphorylation of numerous cellular proteins [44]. A meta-analysis of 7 studies concluded that EGFR expression correlates with decreased survival in gastric cancer [45]. In addition, preclinical data suggest that a subset of gastric cancers (20\%) with EGFR amplification and overexpression can respond to cetuximab therapy [46]. Cetuximab is a monoclonal antibody directed against the EGFR receptor and was shown to improve outcomes in kras wild-type metastatic colorectal cancer [47]. The EXPAND study was a phase III trial which studied capecitabine and cisplatin with or without cetuximab in the first line setting in advanced gastric cancer [48]. Median PFS for chemotherapy/cetuximab was 4.4 months versus 5.6 months for those who received chemotherapy alone $(\mathrm{HR}=1.09$, 95\% CI $0.92-$ $1.29, P=0.32$ ). Adding cetuximab to this chemotherapy combination also did not improve overall survival $(9.4$ months in both arms, $\mathrm{HR}=1.0,95 \%$ CI $0.87-1.17, P=$ 0.95). The phase III REAL-3 trial compared EOX (epirubicin, oxaliplatin, and capecitabine) with or without panitumumab, a fully human antibody targeting the EGFR receptor [49]. Median overall survival in the chemotherapy group was 11.3 months compared to chemotherapy + panitumumab which was 8.8 months $(\mathrm{HR}=1.37,95 \% \mathrm{CI} 1.07-1.76, P=0.013)$. An additional EGFR-targeting drug matuzumab also gave disappointing results in advanced esophagogastric cancer [50]. A combination of ECX chemotherapy/matuzumab failed to improve overall survival (9.4 months for matuzumab group compared with 12.2 months, $\mathrm{HR}=1.02$, 95\% CI 0.61-1.70, $P=0.945)$.
While these results may highlight the lack of importance of the EGFR pathway in esophagogastric cancer, it is important to note that many negative studies were conducted in an unselected population which may explain their negative results.

5.3. C-MET. C-MET has been proposed as a promising new target in advanced disease and a number of phase III trials are now in progress combining MET inhibitors with chemotherapy in the first-line setting for metastatic gastroesophageal cancer. C-MET is a receptor tyrosine kinase which interacts with its ligand HGF (hepatocyte growth factor) [51]. It has been found to be dysregulated in gastric cancers and is involved with tumor proliferation, invasion, and angiogenesis and has antiapoptotic functions in cancer cells [52, 53]. Tumors which harbor high C-MET expression are more likely to have poor survival rates [54]. In a recently reported phase II study, the anti-HGF monoclonal antibody rilotumumab was combined with chemotherapy with progression-free survival (PFS) as the primary endpoint. PFS was 5.7 months in rilotumumab treatment arms versus 4.2 months in the placebo group $(\mathrm{HR}=0.60,80 \%$ CI $0.45-0.79 ; P=0.016)$ [55]. Unfortunately a significant increase in overall survival was not reported although a phase III study is ongoing comparing rilotumumab alone or in combination with cisplatin/capecitabine in the first-line setting [56]. Tivantinib, although originally regarded as a c-MET inhibitor, has been shown to function independently of the c-MET pathway. Studies on lung cancer cell lines have shown that tivantinib does not inhibit cellular MET activity or downstream phosphorylation of Akt or ERK 1/2 in met-dependent cell lines [57]. Another preclinical study has shown that tivantinib inhibits microtubule polymerization independent of c-MET [58]. However, tivantinib has shown promising efficacy as a single agent in a phase II study meriting further study in combination with chemotherapy in a phase III design [59].

\section{Antiangiogenic Therapy}

Angiogenesis is largely recognized as an important aspect of tumorigenesis and preliminary clinical studies suggested a clinical benefit in the addition of bevacizumab, a monoclonal antibody against VEGF-A, in combination with chemotherapy in gastric cancer $[60,61]$. The failure of bevacizumab to improve overall survival in the phase III AVAGAST trial was a disappointing development although interestingly it did appear from a subset analysis that a western population may derive some benefit $[62,63]$. When subset analyses were performed in the AVAGAST trial, it appeared that those with type 3 (distal nondiffuse) gastric cancer and those from European/American populations derived more benefit from bevacizumab than other gastric cancer subtypes or patients from Asian/Pacific populations. The VEGFR-2 (vascular endothelial growth factor receptor-2) antagonist ramucirumab, as reported in the REGARD trial, demonstrated modest activity in patients with advanced gastric or gastroesophageal junction adenocarcinoma who had disease progression after first-line platinum-containing or fluoropyrimidine-containing chemotherapy [64]. Median 
overall survival was 5.2 months (IQR 2.3-9.9) in patients in the ramucirumab group and 3.8 months (IQR 1.7-7.1) in those in the placebo group ( $\mathrm{HR}=0.776,95 \%$ CI $0.603-$ 0.998; $P=0.047$ ). The subsequently reported RAINBOW trial investigated paclitaxel \pm ramucirumab in patients with metastatic GEJ or gastric adenocarcinoma who had disease progression on or within 4 months after first-line platinumand fluoropyrimidine-based combination therapy [65]. The primary endpoint was overall survival (OS). Median OS was 9.63 months for ramucirumab + paclitaxel compared to 7.36 months for paclitaxel alone (HR $=0.807,95 \%$ CI $0.678-$ $0.962, P=0.017)$. Based on these results the combination of ramucirumab + paclitaxel is expected to become a standard of care treatment regimen in the second-line setting for metastatic upper GI tumors. The success of ramucirumab in the 2nd line setting has prompted its clinical investigation in the first line setting. When ramucirumab was combined with FOLFOX in the first-line setting, it did not improve median progression-free survival (6.4 versus 6.7 months, $\mathrm{HR}=0.98$, $95 \%$ CI $0.69-1.37, P=0.89$ ) or overall survival (11.7 versus 11.5 months, HR $=1.08,95 \%$ CI $0.73-1.58$ ) in patients with advanced gastric/GE junction tumors [66]. Clinical trials investigating alternative combinations of chemotherapy with ramucirumab in the first-line setting are ongoing.

Prior to the success of ramucirumab, tyrosine kinase inhibitors had been explored in their roles as antiangiogenic agents in the treatment of esophagogastric tumors. Several tyrosine kinase inhibitors with known antiangiogenic activity are already in use for other tumor types, for example, renal cell cancer. Sunitinib was studied in a phase II trial in patients with advanced gastric or GE junction tumors who had progressed on chemotherapy [67]. The clinical benefit rate was $7.7 \%$ with $32.1 \%$ of patients exhibiting disease stability. By intent-to-treat analysis, median overall survival was 6.8 months (95\% CI 4.4-9.7 months). The addition of docetaxel to sunitinib resulted in a higher objective response rate $(41.1 \%$ versus $14.3 \%, P=0.02$ ) although this study did not meet its primary endpoint of prolonging time-to-progression [68]. Sorafenib, a multitarget TKI of BRAF, VEGF, and PDGFR, was combined with oxaliplatin in a phase II trial in patients who had progressed on first-line cisplatin/fluoropyrimidine chemotherapy [69]. This study did not meet its primary endpoint of efficacy and showed median PFS of 3 months (95\% CI 2.3-4.1 months) and median overall survival of 6.5 months (95\% CI 5.2-9.6 months).

\section{Cancer Cell Stemness}

The characteristic of cancer cells' ability to grow indefinitely has led to the theory that they share common underlying mechanisms with stem cells [70]. BB1608 is an orally available cancer cell stemness inhibitor whose exact target has not been elucidated [71]. It has been shown to inhibit the Stat3, $\beta$-catenin, and Nanog pathways. To date, a phase Ib study has been conducted combining BB1608 and paclitaxel in advanced cancers and of 5 patients enrolled with refractory gastric/GEJ tumors, 2 had partial responses, 1 had stable disease with $25 \%$ regression, and 2 had prolonged disease stability $\geq 24$ weeks.

\section{Hedgehog Inhibitors}

The sonic hedgehog (SHH) pathway is crucial for normal cell differentiation and aberrant function affects gastric cell proliferation, migration, and invasion [72]. A phase II trial combined vismodegib (SHH pathway inhibitor) with FOLFOX in patients with advanced gastric and GE junction tumors [73]. Patients were treated in the first-line setting and the primary endpoint of median PFS was not met in the intention-to-treat population (11.5 months for FOLFOX/vismodegib versus 9.3 months for FOLFOX alone, 95\% CI 8.5-14.4, $P=0.34$ ). However, the expression of CD44, a gastric cancer stem cell marker, was associated with improved survival in the group who received the SHH inhibitor suggesting that $\mathrm{SHH}$ inhibition may only be effective in those with high CD44 expression [74].

\section{IGFR Targeted Therapy}

Insulin-like growth factors (IGF) are potent hormones which regulate cell proliferation, differentiation, and survival via endocrine, paracrine, and autocrine pathways [75]. Increased IGF-1R signaling, a ubiquitously expressed tyrosine kinase receptor, is associated with downstream activation of MAPK/PI3K and is supportive for tumor growth [76]. Cixutumumab, a fully human IgG1 monoclonal antibody specifically targeting IGF-1R, has shown promising antiproliferative activity in vitro and clinically, in soft tissue sarcomas [77]. Cixutumumab was combined with paclitaxel in metastatic esophageal/GE junction cancer in a randomized phase II study [78]. The primary endpoint (PFS) was not met with median PFS reaching 2.6 months for paclitaxel alone and 2.3 months for paclitaxel + cixutumumab (90\% CI 2-3.6 months, $P=0.72)$.

\section{Plk-1 Targeted Therapy}

Human polo-like kinase 1 (Plk-1) plays a pivotal role in mitosis in normal and malignant cells and preclinical studies have highlighted the importance of Plk-1 in tumor development $[79,80]$. Plk-1 is overexpressed in gastric tumor tissue and is associated with worse prognosis [81]. Volasertib (BI 6727) is a potent Plk-1 inhibitor which induces cell cycle arrest and apoptosis and was administered in combination with an angiokinase inhibitor Nintedanib (BIBF 1120) in a phase I dose-escalation study. This found that it was well-tolerated and a single patient with gastric cancer achieved a partial response [82].

\section{FGFR Therapy}

Amplification of the FGFR2 (fibroblast growth factor receptor 2) has been associated with tumorigenesis and results in downstream activation of the MAPK/PI3K pathways [83]. The overexpression of members of the FGF family has been associated with tumor progression/metastasis and is associated with poor survival in gastric cancer [84]. There is currently a phase II trial investigating the effects of dovitinib, a dual VEGF, and FGFR inhibitor, in combination with docetaxel as second-line chemotherapy in gastric cancer [85]. 


\section{PI3K/mTOR Therapy}

The PI3K (phosphatidylinositol 3-kinase) pathway usually becomes activated through growth factor stimulation by EGFR, PDGFR, IGFR, or c-Met [86]. Once PI3K is activated, Akt becomes phosphorylated affecting cell cycle progression and angiogenesis and has antiapoptotic effects [87]. The mTOR pathway is a central kinase pathway that increases the production of proteins which regulate key cellular processes including metabolism, angiogenesis, and cell growth [88]. Phosphorylated mTOR, indicating constitutive activation, has been associated with tumor progression and worse prognosis in gastric cancer patients [89]. Genetic alterations affecting the PI3K/Akt/mTOR pathway are frequently found in gastric cancer [90-92]. There are several potential approaches to inhibit aberrant PI3K/Akt/mTOR signaling including specifically targeting a single component of the pathway, for example, mTOR inhibitors, or dual inhibitors, for example, dual PI3K/mTOR inhibitors. A phase Ib trial has been completed combining a PI3K inhibitor BYL719 with a HSP inhibitor AUY922 in metastatic gastric cancer patients whose tumors are HER2 amplified or who harbor a PI3K mutation [93]. Everolimus, an oral mTOR inhibitor, was investigated in advanced gastric cancer in the GRANITE1 study [94]. Patients who had progressed after 1-2 lines of chemotherapy were randomized to receive everolimus or best supportive care (BSC). Median overall survival was 5.4 months with everolimus and 4.3 months with BSC (HR = $0.90,95 \%$ CI $0.75-1.08, P=0.124)$. There are also several ongoing trials comparing everolimus with chemotherapy (paclitaxel, capecitabine, and oxaliplatin) $[95,96]$.

\section{Clinical Trial Design in Gastroesophageal Cancer}

Many of the clinical trials involving molecularly targeted agents in gastroesophageal cancer have had initially promising results which do not persist later in larger phase II/III studies. Numbers of participants in these trials are small relative to other tumor types (e.g., breast or colon cancer) and this may explain, in part, the negative results. However, for the large part, these trials have been conducted in an unselected population. Designing clinical trials based on molecularly selected populations whose tumors overexpress a particular molecular marker, makes it more challenging to accrue, but represents a more meaningful approach. The PANGEA study (personalized antibodies for gastroesophageal adenocarcinoma) is an example of a trial that will prospectively determine whether assigning patients to targeted therapies based on the molecular profiling of their tumors, will impact median overall survival [97]. This innovative study design may represent the future of targeted therapy evaluation in gastroesophageal cancer where a variety of targeted therapies are now available to patients based on the individual molecular biology of their tumor.

\section{Conclusion}

The development of new therapies for advanced gastroesophageal cancer has been slow compared to other common tumors. Upper GI tumors represent diverse and heterogeneous diseases with multiple etiological factors including viral and bacterial infection via EBV and Helicobacter pylori, inherited familial syndromes, and other risk factors including diet, smoking, and alcohol consumption. Taken together, upper GI tumors are second only to lung cancer in terms of cancer related morbidity. In recent years incremental breakthroughs in our understanding of the molecular biology underlying the development of these cancers have taken place and these findings are now being translated into clinical research. High density genomic profiling arrays analyzing somatic copy-number alterations in gastroesophageal cancers have identified amplified genes in 37\% of tumors, most notably, ERBB2, FGFR1, FGFR2, EGFR, and MET [27]. Until recently however the "one-size fits all approach" to enrolling patients on clinical trials has failed in gastroesophageal cancer as it has in other tumor types.

The Cancer Genome Atlas Research Network has recently identified other viable targets and it appears likely that the four molecular subtypes of gastric cancer described in this study should advance clinical research in the years to come. In addition to targeted agents there has been a rapid expansion in our understanding of the immune environment changes that occur in these tumors allowing them to avoid immuneediting. Future studies may combine targeted therapeutics with immunotherapies such as checkpoint inhibitors. Patient selection should be performed in all clinical trials to ensure that molecularly targeted therapies can fulfill their promise in upper GI tumors.

\section{Conflict of Interests}

The authors declare that there is no conflict of interests regarding the publication of this paper.

\section{References}

[1] R. Siegel, J. Ma, Z. Zou, and A. Jemal, "Cancer statistics, 2014," CA: Cancer Journal for Clinicians, vol. 64, no. 1, pp. 9-29, 2014.

[2] J. Ferlay, H.-R. Shin, F. Bray, D. Forman, C. Mathers, and D. M. Parkin, "Estimates of worldwide burden of cancer in 2008: GLOBOCAN 2008," International Journal of Cancer, vol. 127, no. 12, pp. 2893-2917, 2010.

[3] K. D. Crew and A. I. Neugut, "Epidemiology of upper gastrointestinal malignancies," Seminars in Oncology, vol. 31, no. 4, pp. 450-464, 2004.

[4] D. Forman, "An international association between Helicobacter pylori infection and gastric cancer. The EUROGAST Study Group," The Lancet, vol. 341, no. 8857, pp. 1359-1362, 1993.

[5] J. H. Rubenstein and J. B. Taylor, "Meta-analysis: the association of oesophageal adenocarcinoma with symptoms of gastrooesophageal reflux," Alimentary Pharmacology and Therapeutics, vol. 32, no. 10, pp. 1222-1227, 2010.

[6] H. Hampel, N. S. Abraham, and H. B. El-Serag, "Meta-analysis: obesity and the risk for gastroesophageal reflux disease and its complications," Annals of Internal Medicine, vol. 143, no. 3, pp. 199-211, 2005.

[7] D. Cunningham, N. Starling, S. Rao et al., "Capecitabine and oxaliplatin for advanced esophagogastric cancer," The New England Journal of Medicine, vol. 358, no. 1, pp. 36-46, 2008. 
[8] M. J. Overman, S. M. Kazmi, J. Jhamb et al., "Weekly docetaxel, cisplatin, and 5-fluorouracil as initial therapy for patients with advanced gastric and esophageal cancer," Cancer, vol. 116, no. 6, pp. 1446-1453, 2010

[9] N. C. Tebbutt, M. M. Cummins, T. Sourjina et al., "Randomised, non-comparative phase II study of weekly docetaxel with cisplatin and 5-fluorouracil or with capecitabine in oesophagogastric cancer: the AGITG ATTAX trial," British Journal of Cancer, vol. 102, no. 3, pp. 475-481, 2010.

[10] M. A. Shah, S. Shibata, R. G. Stoller et al., "Random assignment multicenter phase II study of modified docetaxel, cisplatin, fluorouracil (mDCF) versus DCF with growth factor support (GCSF) in metastatic gastroesophageal adenocarcinoma (GE)," in ASCO Meeting Abstracts, vol. 28, p. 4014, 2014.

[11] H. E. R. Ford, A. Marshall, J. A. Bridgewater et al., "Docetaxel versus active symptom control for refractory oesophagogastric adenocarcinoma (COUGAR-02): an open-label, phase 3 randomised controlled trial," The Lancet Oncology, vol. 15, no. 1, pp. 78-86, 2014.

[12] H. S. Kim, H. J. Kim, S. Y. Kim et al., "Second-line chemotherapy versus supportive cancertreatment in advanced gastric cancer: a meta-analysis," Annals of Oncology, vol. 24, no. 11, pp. 28502854, 2013.

[13] E. Hawkes, A. F. C. Okines, D. Papamichael et al., "Docetaxel and irinotecan as second-line therapy for advanced oesophagogastric cancer," European Journal of Cancer, vol. 47, no. 8, pp. 1146-1151, 2011.

[14] S. Hironaka, S. Zenda, N. Boku, A. Fukutomi, T. Yoshino, and Y. Onozawa, "Weekly paclitaxel as second-line chemotherapy for advanced or recurrent gastric cancer," Gastric Cancer, vol. 9, no. 1, pp. 14-18, 2006.

[15] A. Boussioutas, H. Li, J. Liu et al., "Distinctive patterns of gene expression in premalignant gastric mucosa and gastric cancer," Cancer Research, vol. 63, no. 10, pp. 2569-2577, 2003.

[16] I. B. Tan, T. Ivanova, K. H. Lim et al., "Intrinsic subtypes of gastric cancer, based on gene expression pattern, predict survival and respond differently to chemotherapy," Gastroenterology, vol. 141, no. 2, pp. 476-485, 485 el-11, 2011.

[17] Z. Lei, I. B. Tan, K. Das et al., "Identification of molecular subtypes of gastric cancer with different responses to pi3-kinase inhibitors and 5-fluorouracil," Gastroenterology, vol. 145, no. 3, pp. 554-565, 2013.

[18] The Cancer Genome Atlas Research Network, "Comprehensive molecular characterization of gastric adenocarcinoma," Nature, vol. 513, no. 7517, pp. 202-209, 2014.

[19] J. Gu, J. A. Ajani, E. T. Hawk et al., "Genome-wide catalogue of chromosomal aberrations in Barrett's esophagus and esophageal adenocarcinoma: a high-density single nucleotide polymorphism array analysis," Cancer Prevention Research, vol. 3, no. 9, pp. 1176-1186, 2010.

[20] A. Varis, P. Puolakkainen, H. Savolainen et al., "DNA copy number profiling in esophageal Barrett adenocarcinoma: comparison with gastric adenocarcinoma and esophageal squamous cell carcinoma," Cancer Genetics and Cytogenetics, vol. 127, no. 1, pp. 53-58, 2001.

[21] B. J. Reid, L. J. Prevo, P. C. Galipeau et al., "Predictors of progression in Barrett's esophagus II: baseline 17p (p53) loss of heterozygosity identifies a patient subset at increased risk for neoplastic progression," The American Journal of Gastroenterology, vol. 96, no. 10, pp. 2839-2848, 2001.
[22] M. C. Hollstein, R. A. Metcalf, J. A. Welsh, R. Montesano, and C. C. Harris, "Frequent mutation of the p53 gene in human esophageal cancer," Proceedings of the National Academy of Sciences of the United States of America, vol. 87, no. 24, pp. 99589961, 1990.

[23] A. G. Casson, T. Mukhopadhyay, K. R. Cleary, J. Y. Ro, B. Levin, and J. A. Roth, "p53 gene mutations in Barrett's epithelium and esophageal cancer," Cancer Research, vol. 51, no. 16, pp. 44954499, 1991.

[24] E. L. Powell, L. M. Leoni, M. I. Canto et al., "Concordant loss of MTAP and p16/CDKN2A expression in gastroesophageal carcinogenesis: evidence of homozygous deletion in esophageal noninvasive precursor lesions and therapeutic implications," The American Journal of Surgical Pathology, vol. 29, no. 11, pp. 1497-1504, 2005.

[25] M. D. Abeloff, J. C. Eggleston, G. Mendelsohn, D. S. Ettinger, and S. B. Baylin, "Changes in morphologic and biochemical characteristics of small cell carcinoma of the lung. A clinicopathologic study," The American Journal of Medicine, vol. 66, no. 5, pp. 757-764, 1979.

[26] N. Agrawal, Y. Jiao, C. Bettegowda et al., "Comparative genomic analysis of esophageal adenocarcinoma and squamous cell carcinoma," Cancer Discovery, vol. 2, no. 10, pp. 899-905, 2012.

[27] A. M. Dulak, S. E. Schumacher, J. van Lieshout et al., "Gastrointestinal adenocarcinomas of the esophagus, stomach, and colon exhibit distinct patterns of genome instability and oncogenesis," Cancer Research, vol. 72, no. 17, pp. 4383-4393, 2012.

[28] M. A. Olayioye, "Update on HER-2 as a target for cancer therapy-intracellular signaling pathways of ErbB2/HER-2 and family members," Breast Cancer Research, vol. 3, no. 6, pp. 385$389,2001$.

[29] M. Tanner, M. Hollmén, T. T. Junttila et al., "Amplification of HER-2 in gastric carcinoma: association with Topoisomerase II $\alpha$ gene amplification, intestinal type, poor prognosis and sensitivity to trastuzumab," Annals of Oncology, vol. 16, no. 2, pp. 273-278, 2005.

[30] D. I. Park, J. W. Yun, J. H. Park et al., "HER-2/neu amplification is an independent prognostic factor in gastric cancer," Digestive Diseases and Sciences, vol. 51, no. 8, pp. 1371-1379, 2006.

[31] Y. Y. Janjigian, D. Werner, C. Pauligk et al., "Prognosis of metastatic gastric and gastroesophageal junction cancer by HER2 status: a European and USA International collaborative analysis," Annals of Oncology, vol. 23, no. 10, pp. 2656-2662, 2012.

[32] C. Gómez-Martin, E. Garralda, M. J. Echarri et al., "HER2/neu testing for anti-HER2-based therapies in patients with unresectable and/or metastatic gastric cancer," Journal of Clinical Pathology, vol. 65, no. 8, pp. 751-757, 2012.

[33] Y.-J. Bang, E. Van Cutsem, A. Feyereislova et al., "Trastuzumab in combination with chemotherapy versus chemotherapy alone for treatment of HER2- positive advanced gastric or gastrooesophageal junction cancer (ToGA): a phase 3, open-label, randomised controlled trial," The Lancet, vol. 376, no. 9742, pp. 687-697, 2010.

[34] A. Goldhirsch, R. D. Gelber, M. J. Piccart-Gebhart et al., "2 years versus 1 year of adjuvant trastuzumab for HER2-positive breast cancer (HERA): an open-label, randomised controlled trial," The Lancet, vol. 382, no. 9897, pp. 1021-1028, 2013.

[35] G. von Minckwitz, K. Schwedler, M. Schmidt et al., "Trastuzumab beyond progression: overall survival analysis of the GBG 26/BIG 3-05 phase III study in HER2-positive breast cancer," European Journal of Cancer, vol. 47, no. 15, pp. 2273-2281, 2011. 
[36] D. Sakai, T. Satoh, Y. Kurokawa et al., "A phase II trial of trastuzumab combined with irinotecan in patients with advanced HER2-positive chemo-refractory gastric cancer: Osaka Gastrointestinal Cancer Chemotherapy Study Group OGSG1203 (HERBIS-5)," Japanese Journal of Clinical Oncology, vol. 43, no. 8, pp. 838-840, 2013.

[37] E. R. Wood, A. T. Truesdale, O. B. McDonald et al., "A unique structure for epidermal growth factor receptor bound to GW572016 (Lapatinib): relationships among protein conformation, inhibitor off-rate, and receptor activity in tumor cells," Cancer Research, vol. 64, no. 18, pp. 6652-6659, 2004.

[38] T. Satoh, R.-H. Xu, H. C. Chung et al., "Lapatinib plus paclitaxel versus paclitaxel alone in the second-line treatment of HER2-amplified advanced gastric cancer in Asian populations: TyTAN-a randomized, phase III study," Journal of Clinical Oncology, vol. 32, no. 19, pp. 2039-2049, 2014.

[39] C. W. Adams, D. E. Allison, K. Flagella et al., "Humanization of a recombinant monoclonal antibody to produce a therapeutic HER dimerization inhibitor, pertuzumab," Cancer Immunology, Immunotherapy, vol. 55, no. 6, pp. 717-727, 2006.

[40] J. Tabernero, P. Marcelo Hoff, L. Shen et al., "Pertuzumab (P) with trastuzumab (T) and chemotherapy (CTX) in patients (pts) with HER2-positive metastatic gastric or gastroesophageal junction (GEJ) cancer: an international phase III study (JACOB)," Journal of Clinical Oncology, 2013, abstr TPS4150.

[41] P. M. LoRusso, D. Weiss, E. Guardino, S. Girish, and M. X. Sliwkowski, "Trastuzumab emtansine: a unique antibody-drug conjugate in development for human epidermal growth factor receptor 2-positive cancer," Clinical Cancer Research, vol. 17, no. 20, pp. 6437-6447, 2011.

[42] H. K. Erickson, P. U. Park, W. C. Widdison et al., "Antibodymaytansinoid conjugates are activated in targeted cancer cells by lysosomal degradation and linker-dependent intracellular processing," Cancer Research, vol. 66, no. 8, pp. 4426-4433, 2006.

[43] UK Clinical Research Network Study Portfolio, "NCRN369: GATSBY TDM1 vs taxane in previously treated advanced HER2+ gastric ca," UKCRN 12204, http://public.ukcrn.org.uk/ search/StudyDetail.aspx?StudyID=12204.

[44] S. K. Muthuswamy, M. Gilman, and J. S. Brugge, "Controlled dimerization of ErbB receptors provides evidence for differential signaling by homo- and heterodimers," Molecular and Cellular Biology, vol. 19, no. 10, pp. 6845-6857, 1999.

[45] C. Chen, J.-M. Yang, T.-T. Hu et al., "Prognostic role of human epidermal growth factor receptor in gastric cancer: a systematic review and meta-analysis," Archives of Medical Research, vol. 44, no. 5, pp. 380-389, 2013.

[46] L. Zhang, J. Yang, J. Cai et al., "A subset of gastric cancers with EGFR amplification and overexpression respond to cetuximab therapy," Scientific Reports, vol. 3, article 2992, 2013.

[47] E. Van Cutsem, C.-H. Köhne, I. Láng et al., "Cetuximab plus irinotecan, fluorouracil, and leucovorin as first-line treatment for metastatic colorectal cancer: updated analysis of overall survival according to tumor KRAS and BRAF mutation status," Journal of Clinical Oncology, vol. 29, no. 15, pp. 2011-2019, 2011.

[48] F. Lordick, Y.-K. Kang, H.-C. Chung et al., "Capecitabine and cisplatin with or without cetuximab for patients with previously untreated advanced gastric cancer (EXPAND): a randomised, open-label phase 3 trial," The Lancet Oncology, vol. 14, no. 6, pp. 490-499, 2013.
[49] T. Waddell, I. Chau, D. Cunningham et al., "Epirubicin, oxaliplatin, and capecitabine with or without panitumumab for patients with previously untreated advanced oesophagogastric cancer (REAL3): a randomised, open-label phase 3 trial," The Lancet Oncology, vol. 14, no. 6, pp. 481-489, 2013.

[50] S. Rao, N. Starling, D. Cunningham et al., "Matuzumab plus epirubicin, cisplatin and capecitabine (ECX) compared with epirubicin, cisplatin and capecitabine alone as first-line treatment in patients with advanced oesophago-gastric cancer: a randomised, multicentre open-label phase II study," Annals of Oncology, vol. 21, no. 11, pp. 2213-2219, 2010.

[51] T. Inoue, H. Kataoka, K. Goto et al., "Activation of c-Met (hepatocyte growth factor receptor) in human gastric cancer tissue," Cancer Science, vol. 95, no. 10, pp. 803-808, 2004.

[52] P. Conrotto, D. Valdembri, S. Corso et al., "Sema4D induces angiogenesis through Met recruitment by Plexin B1," Blood, vol. 105, no. 11, pp. 4321-4329, 2005.

[53] T.-J. Huang, J.-Y. Wang, S.-R. Lin, S.-T. Lian, and J.-S. Hsieh, "Overexpression of the c-met protooncogene in human gastric carcinoma-correlation to clinical features," Acta Oncologica, vol. 40, no. 5, pp. 638-643, 2001.

[54] S. Yu, Y. Yu, N. Zhao, J. Cui, W. Li, and T. Liu, "c-Met as a prognostic marker in gastric cancer: a systematic review and meta-analysis," PLoS ONE, vol. 8, no. 11, Article ID e79137, 2013.

[55] T. Iveson, R. C. Donehower, I. Davidenko et al., "Rilotumumab in combination with epirubicin, cisplatin, and capecitabine as first-line treatment for gastric or oesophagogastric junction adenocarcinoma: an open-label, dose de-escalation phase $1 \mathrm{~b}$ study and a double-blind, randomised phase 2 study," The Lancet Oncology, vol. 15, no. 9, pp. 1007-1018, 2014.

[56] A Phase 3 Study of Rilotumumab (AMG 102) with Cisplatin and Capecitabine (CX) as First-line Therapy in Gastric Cancer (RILOMET-2), NCT02137343, 2014, http://clinicaltrials.gov/ show/NCT02137343.

[57] A. Calles, N. Kwiatkowski, B. K. Cammarata, D. Ercan, N. S. Gray, and P. A. Jänne, "Tivantinib (ARQ 197) efficacy is independent of MET inhibition in non-small-cell lung cancer cell lines," Molecular Oncology, vol. 9, no. 1, pp. 260-269, 2015.

[58] R. Katayama, A. Aoyama, T. Yamori et al., "Cytotoxic activity of tivantinib (ARQ 197) is not due solely to c-MET inhibition," Cancer Research, vol. 73, no. 10, pp. 3087-3096, 2013.

[59] Y.-K. Kang, K. Muro, M.-H. Ryu et al., "A phase II trial of a selective c-Met inhibitor tivantinib (ARQ 197) monotherapy as a second- or third-line therapy in the patients with metastatic gastric cancer," Investigational New Drugs, vol. 32, no. 2, pp. 355361, 2014.

[60] N. Ferrara, H.-P. Gerber, and J. LeCouter, "The biology of VEGF and its receptors," Nature Medicine, vol. 9, no. 6, pp. 669-676, 2003.

[61] M. A. Shah, R. K. Ramanathan, D. H. Ilson et al., "Multicenter phase II study of irinotecan, cisplatin, and bevacizumab in patients with metastatic gastric or gastroesophageal junction adenocarcinoma," Journal of Clinical Oncology, vol. 24, no. 33, pp. 5201-5206, 2006.

[62] M. A. Shah, E. Van Cutsem, Y. K. Kang et al., "Survival analysis according to disease subtype in AVAGAST: first-line capecitabine and cisplatin plus bevacizumab (bev) or placebo in patients (pts) with advanced gastric cancer," Journal of Clinical Oncology, vol. 30, supplement 4, abstract 5, 2012. 
[63] A. Ohtsu, M. A. Shah, E. van Cutsem et al., "Bevacizumab in combination with chemotherapy as first-line therapy in advanced gastric cancer: a randomized, double-blind, placebocontrolled phase III study," Journal of Clinical Oncology, vol. 29, no. 30, pp. 3968-3976, 2011.

[64] C. S. Fuchs, J. Tomasek, C. J. Yong et al., "Ramucirumab monotherapy for previously treated advanced gastric or gastrooesophageal junction adenocarcinoma (REGARD): an international, randomised, multicentre, placebo-controlled, phase 3 trial," The Lancet, vol. 383, no. 9911, pp. 31-39, 2014.

[65] H. Wilke, E. van Cutsem, S. C. Oh et al., "RAINBOW: a global, phase III, randomized, double-blind study of ramucirumab plus paclitaxel versus placebo plus paclitaxel in the treatment of metastatic gastroesophageal junction (GEJ) and gastric adenocarcinoma following disease progression on firstline platinum- and fluoropyrimidine-containing combination therapy rainbow IMCL CP12-0922 (I4T-IE-JVBE)," Journal of Clinical Oncology, vol. 32, supplement 3, 2014, Gastrointestinal Cancers Symposium abstract no. LBA7.

[66] H. H. Yoon, J. C. Bendell, F. S. Braiteh et al., "Ramucirumab (RAM) plus FOLFOX as front-line therapy (Rx) for advanced gastric or esophageal adenocarcinoma (GE-AC): randomized, double-blind, multicenter phase 2 trial," Journal of Clinical Oncology, vol. 32, no. 5s, 2014, abstr 4004.

[67] Y.-J. Bang, Y.-K. Kang, W. K. Kang et al., "Phase II study of sunitinib as second-line treatment for advanced gastric cancer," Investigational New Drugs, vol. 29, no. 6, pp. 1449-1458, 2011.

[68] J. H. Yi, J. Lee, J. Lee et al., "Randomised phase II trial of docetaxel and sunitinib in patients with metastatic gastric cancer who were previously treated with fluoropyrimidine and platinum," British Journal of Cancer, vol. 106, no. 9, pp. 14691474, 2012.

[69] M. Martin-Richard, R. Gallego, C. Pericay et al., "Multicenter phase II study of oxaliplatin and sorafenib in advanced gastric adenocarcinoma after failure of cisplatin and fluoropyrimidine treatment. A GEMCAD study," Investigational New Drugs, vol. 31, no. 6, pp. 1573-1579, 2013.

[70] D. J. Wong, E. Segal, and H. Y. Chang, "Stemness, cancer and cancer stem cells," Cell Cycle, vol. 7, no. 23, pp. 3622-3624, 2008.

[71] M. Hitron, J. Stephenson, K. N. Chi et al., "A phase 1b study of the cancer stem cell inhibitor BBI608 administered with paclitaxel in patients with advanced malignancies," Journal of Clinical Oncology, vol. 32, supplement, abstract 2530, 2014.

[72] R. Bai, H. Zhao, X. Zhang, and S. Du, "Characterization of sonic hedgehog inhibition in gastric carcinoma cells," Oncology Letters, vol. 7, no. 5, pp. 1381-1384, 2014.

[73] D. J. Cohen, P. J. Christos, J. A. Sparano et al., "A randomized phase II study of vismodegib (V), a hedgehog (HH) pathway inhibitor, combined with FOLFOX in patients (pts) with advanced gastric and gastroesophageal junction (GEJ) carcinoma: a New York Cancer Consortium led study," Journal of Clinical Oncology, vol. 31, supplement 4, abstract 67, 2013.

[74] C. Yoon, D. J. Park, B. Schmidt et al., "CD44 expression denotes a subpopulation of gastric cancer cells in which Hedgehog signaling promotes chemotherapy resistance," Clinical Cancer Research, vol. 20, no. 15, pp. 3974-3988, 2014.

[75] K. Pavelić, T. Kolak, S. Kapitanović et al., "Gastric cancer: the role of insulin-like growth factor 2 (IGF 2) and its receptors (IGF IR and M6-P/IGF 2R)," The Journal of Pathology, vol. 201, no. 3, pp. $430-438,2003$.
[76] J. Gao, Y. S. Chang, B. Jallal, and J. Viner, “Targeting the insulinlike growth factor axis for the development of novel therapeutics in oncology," Cancer Research, vol. 72, no. 1, pp. 3-12, 2012.

[77] P. Schöffski, D. Adkins, J.-Y. Blay et al., "An open-label, phase 2 study evaluating the efficacy and safety of the anti-IGF$1 \mathrm{R}$ antibody cixutumumab in patients with previously treated advanced or metastatic soft-tissue sarcoma or Ewing family of tumours," European Journal of Cancer, vol. 49, no. 15, pp. 32193228, 2013.

[78] S. J. Cohen, Y. Feng, P. J. Catalano et al., "E2208: randomized phase II study of paclitaxel with or without the anti-IGF-IR antibody cixutumumab (IMC-A12) as second-line treatment for patients with metastatic esophageal or GE junction cancer," Journal of Clinical Oncology, vol. 32, no. 5, supplement, 2014, ASCO Annual Meeting abstract no. 4020.

[79] F. A. Barr, H. H. W. Silljé, and E. A. Nigg, "Polo-like kinases and the orchestration of cell division," Nature Reviews Molecular Cell Biology, vol. 5, no. 6, pp. 429-440, 2004.

[80] B. Spänkuch-Schmitt, G. Wolf, C. Solbach et al., "Downregulation of human polo-like kinase activity by antisense oligonucleotides induces growth inhibition in cancer cells," Oncogene, vol. 21, no. 20, pp. 3162-3171, 2002.

[81] W. Weichert, A. Ullrich, M. Schmidt et al., "Expression patterns of polo-like kinase 1 in human gastric cancer," Cancer Science, vol. 97, no. 4, pp. 271-276, 2006.

[82] F. G. de Braud, S. Cascinu, G. Spitaleri et al., "A phase I dose-escalation study of volasertib (BI 6727) combined with nintedanib (BIBF 1120) in advanced solid tumors," Journal of Clinical Oncology, vol. 31, supplement, abstract 2556, 2013.

[83] M. Katoh, "FGF signaling network in the gastrointestinal tract (review)," International Journal of Oncology, vol. 29, no. 1, pp. 163-168, 2006.

[84] H. Murase, M. Inokuchi, Y. Takagi, K. Kato, K. Kojima, and K. Sugihara, "Prognostic significance of the co-overexpression of fibroblast growth factor receptors 1, 2 and 4 in gastric cancer," Molecular and Clinical Oncology, vol. 2, no. 4, pp. 509-517, 2014.

[85] Dovitinib for Gastric Cancer With FGFR2 Amplification, NCT01719549, 2014, http://www.clinicaltrials.gov/ct2/show/ NCT01719549?term=fgf+gastric\&rank=1.

[86] J. A. Fresno Vara, E. Casado, J. de Castro, P. Cejas, C. BeldaIniesta, and M. González-Barón, "PI3K/Akt signalling pathway and cancer," Cancer Treatment Reviews, vol. 30, no. 2, pp. 193204, 2004.

[87] B. D. Manning and L. C. Cantley, "AKT/PKB signaling: navigating downstream," Cell, vol. 129, no. 7, pp. 1261-1274, 2007.

[88] D. C. Fingar, C. J. Richardson, A. R. Tee, L. Cheatham, C. Tsou, and J. Blenis, "mTOR controls cell cycle progression through its cell growth effectors S6K1 and 4E-BP1/eukaryotic translation initiation factor 4E," Molecular and Cellular Biology, vol. 24, no. 1, pp. 200-216, 2004.

[89] T. Murayama, M. Inokuchi, Y. Takagi et al., "Relation between outcomes and localisation of p-mTOR expression in gastric cancer," British Journal of Cancer, vol. 100, no. 5, pp. 782-788, 2009.

[90] S. Velho, C. Oliveira, A. Ferreira et al., "The prevalence of PIK3CA mutations in gastric and colon cancer," European Journal of Cancer, vol. 41, no. 11, pp. 1649-1654, 2005.

[91] Y. Sukawa, H. Yamamoto, K. Nosho et al., "Alterations in the human epidermal growth factor receptor 2-phosphatidylinositol 3-kinase-v-Akt pathway in gastric cancer," World Journal of Gastroenterology, vol. 18, no. 45, pp. 6577-6586, 2012. 
[92] M. Li, H. Sun, L. Song, X. Gao, W. Chang, and X. Qin, "Immunohistochemical expression of mTOR negatively correlates with PTEN expression in gastric carcinoma," Oncology Letters, vol. 4, no. 6, pp. 1213-1218, 2012.

[93] PI3K Inhibitor BYL719 in Combination With the HSP90 Inhibitor AUY922 in Patients With Advanced or Metastatic Gastric Cancer, NCT01613950, 2014, http://clinicaltrials.gov/ show/NCT01613950.

[94] A. Ohtsu, J. A. Ajani, Y.-X. Bai et al., "Everolimus for previously treated advanced gastric cancer: results of the randomized, double-blind, phase III GRANITE-1 study," Journal of Clinical Oncology, vol. 31, no. 31, pp. 3935-3943, 2013.

[95] RAD001 in Combination With Capecitabine and Oxaliplatin (XELOX) in Patients With Advanced Gastric Cancer, NCT01049620, 2014, http://clinicaltrials.gov/show/NCT01613950.

[96] A Randomized, Double Blind Study Evaluating Paclitaxel with and without RAD001 in Patients with Gastric Carcinoma After Prior Chemotherapy (AIO-STO-0111), NCT01248403, 2014, http://clinicaltrials.gov/show/NCT01248403.

[97] PANGEA-IMBBP: Personalized Antibodies for Gastro-Esophageal Adenocarcinoma-A 1st Pilot Metastatic Trial of Biologics Beyond Progression, NCT02213289, 2014, http://clinicaltrials.gov/show/NCT02213289. 


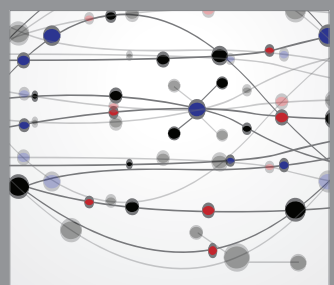

The Scientific World Journal
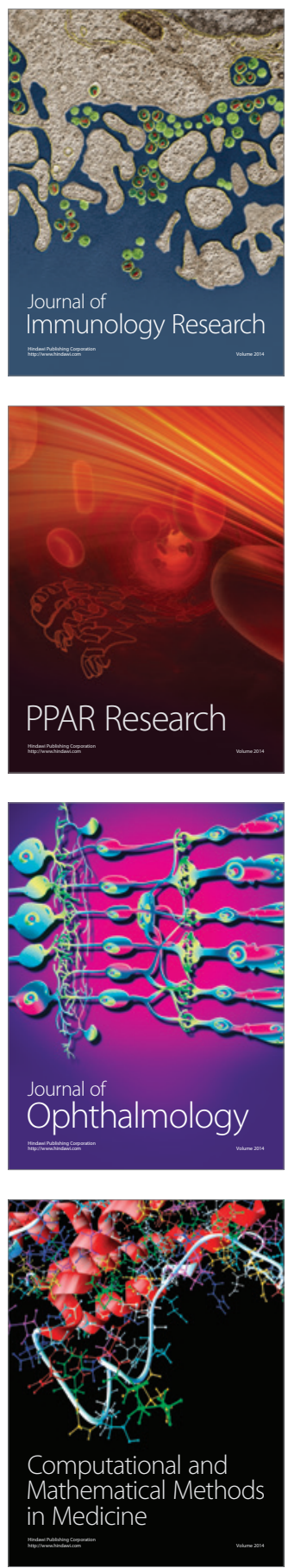

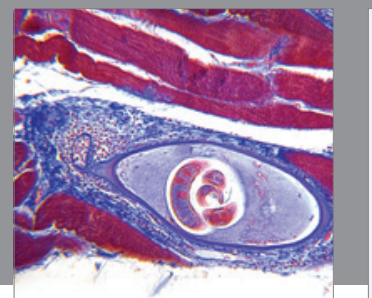

Gastroenterology

Research and Practice
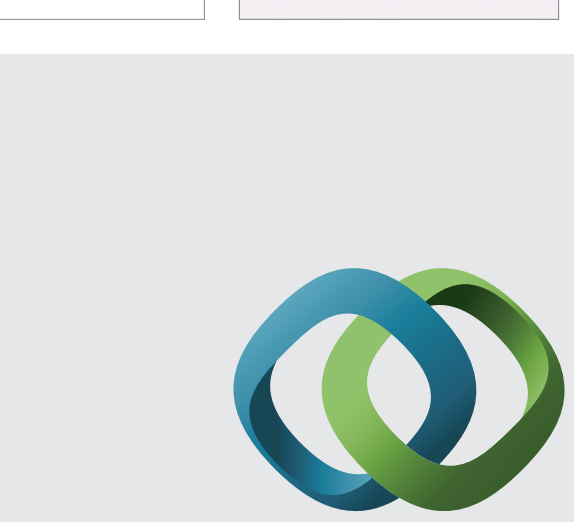

\section{Hindawi}

Submit your manuscripts at

http://www.hindawi.com
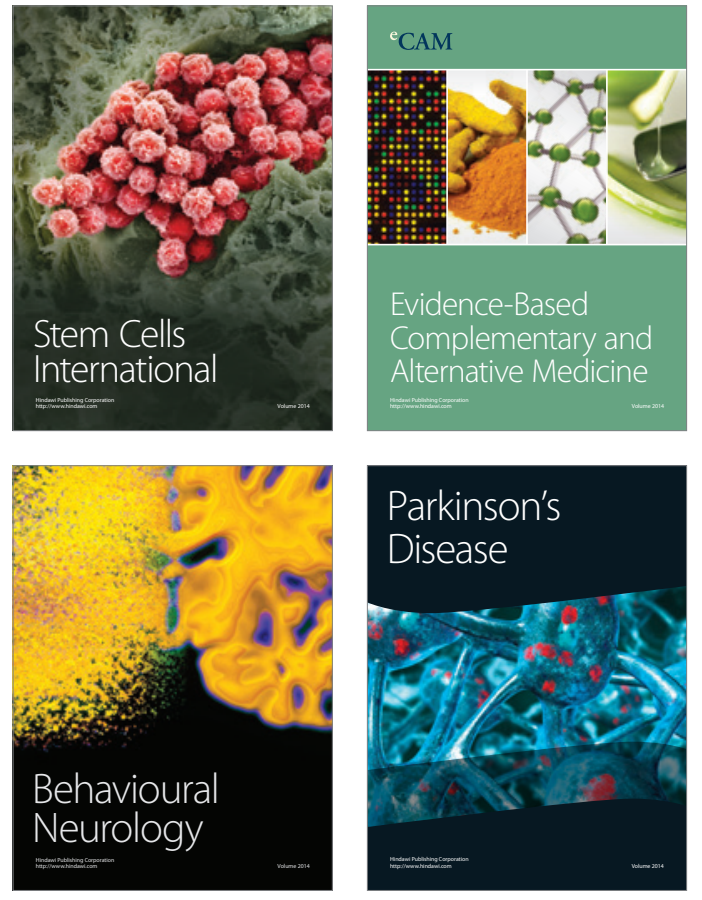
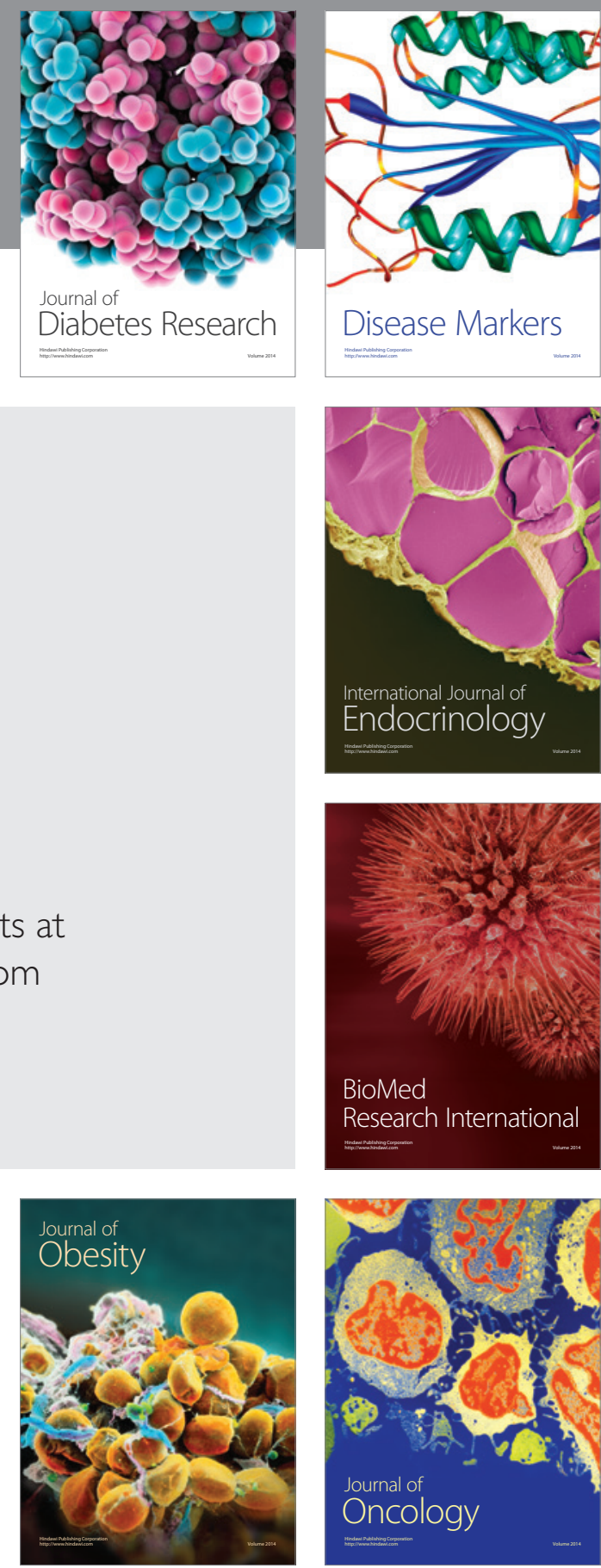

Disease Markers
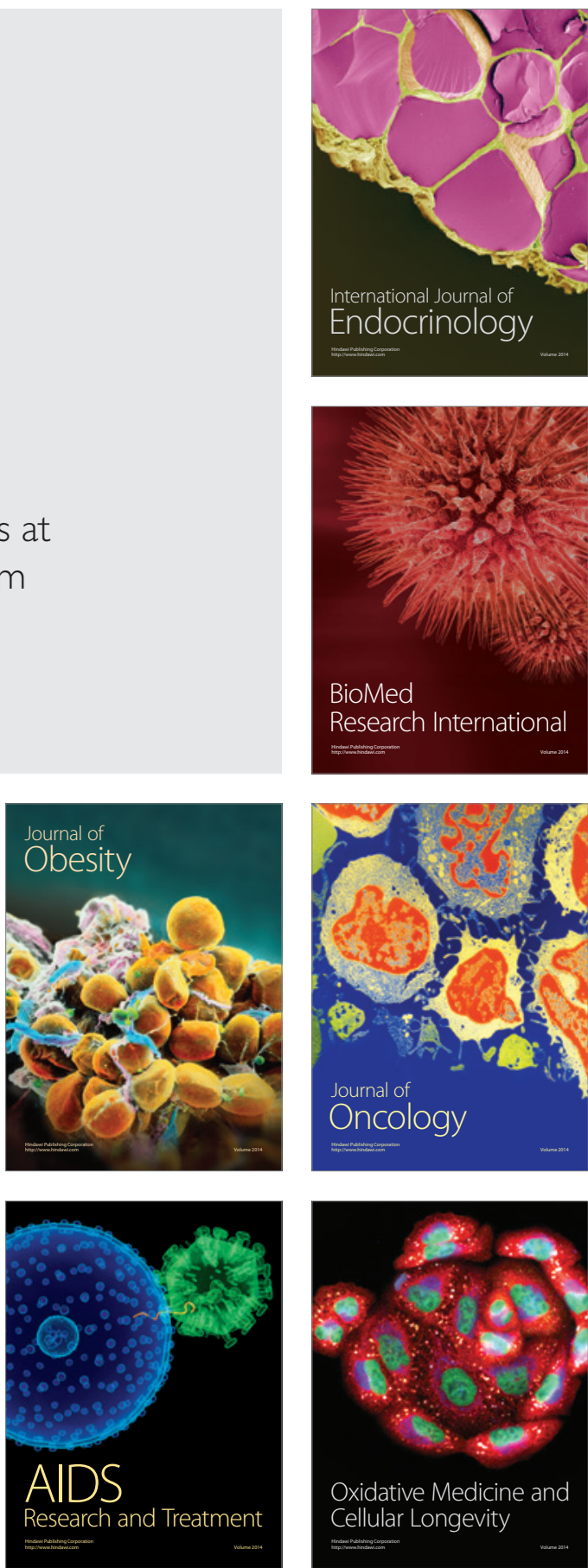